\title{
Analyzing the Tropospheric Delay Estimates on Global Navigation Satellite Systems (GNSS) with Precise Point Positioning (PPP) Services using the goGPS software.
}

\author{
Syachrul Arief ${ }^{1}$ and Andrea Gatti ${ }^{2}$ \\ ${ }^{1}$ Geospatial Information Agency, Indonesia \\ ${ }^{2}$ Geomatics Research and Development s.r.l. - Lomazzo, Italy
}

Corresponding author: Syachrul Arief | Email: syachrul.arief@big.go.id

Received: 13/05/2020 Revised: 09/08/2020 Accepted: 08/09/2020

\begin{abstract}
The tropospheric delay is an essential source of error for positioning using the Global Navigation Satellite System (GNSS). Scientific applications of GNSS positioning such as the study of earth crust deformation and earthquake prediction require high accuracy in positioning, an analysis of tropospheric delay calculations is needed to improve the accuracy of GNSS positioning. One part of the tropospheric delay is Zenith tropospheric delays (ZTD), which are estimated using the Precise Point Positioning (PPP) method. ZTD estimates can be beneficial for meteorological applications, for example, is the estimation of water vapor levels in the atmosphere from the estimated ZTD. We use GNSS data from the BAKO station in Cibinong and JOG2 station located in Yogyakarta. The GNSS data format is an Independent Exchange Receiver (RINEX), which we extracted using the sophisticated open-source GNSS software, called goGPS version 1.0 Beta from Geomatics Research and Development s.r.l. - Lomazzo, Italy. We validate the results of the extraction process with two international tropospheric products from International GNSS Services (IGS) with commercial software Bernese version 5 and the University of Nevada Reno (UNR) with software from NASA Jet Propulsion Laboratory (JPL) namely GIPSY / OASIS II. Epoch in this study, we use days of the year (DOY) 022-025 / 22-25 January representing the rainy season and DOY $230-233$ to coincide on August 17-20 representing the dry season 2018. Our results obtained ZTD values both in January and August, and the two BAKO and JOG2 stations were consistent and worked well at different times and stations. RMS throughout DOY, both at BAKO and JOG2 stations, show small values $<2 \mathrm{~mm}$. The RMS value is relatively small, meaning that the troposphere estimation process with goGPS shows a good agreement because it is almost the same as the international troposphere products from UNR and IGS. This means that the ZTD estimation process from goGPS software can be an alternative to paid software. The range of ZTD values in January tends to be higher than in August, meaning the value of ZTD has a strong correlation with changes in the rainy and dry seasons, this shows that ZTD can be useful for meteorological purposes.
\end{abstract}

Keywords: Tropospheric Delay, GNSS, PPP, goGPS.

(C) Author(s) 2020. This is an open access article under the Creative Commons Attribution-ShareAlike 4.0 International License (CC BY-SA 4.0).

\section{Introduction}

GPS will work perfectly if there are no obstacles that interfere with the signal sent by satellite. Though, in reality, there are many mistakes involved in GPS. These errors can be divided into three categories, space segment errors, user segment errors, and atmospheric errors.

1.1. Space segment errors

Space segment errors consist of two types of errors, and one is a satellite ephemeris error, the other is a satellite clock error. Satellite ephemeris errors occur due to inaccuracies in satellite orbit. This orbital error can be decomposed into three components along a satellite orbit, namely radial, along-track and cross-track. The error in the radial direction is small compared to the error along the trajectory and cross-lane, which is fortunate because the error of measuring the apparent range is in the same direction as the radial error component, the line between the satellite and the receiver. The Kalman filter is used to derive ephemeris parameters (Enge \& Misra, 2001). The measurement carried out by the GPS receiver is the transit time of the signal between the satellite and the receiver, which is determined by the difference between the signal transmission time from the satellite and the reception time measured by the receiver (Enge \& Misra, 2006). Therefore, the accuracy of the clock is essential, and mistakes must be avoided. An error of $1 \mu$ s can cause an offset of about $300 \mathrm{~m}$ because the travel time is multiplied by the speed of light to get the distance between the satellite and the receiver (Nicholas Zinas, 2016). To keep the satellite clock error as small as possible, each GPS 
satellite carries an atomic clock. The atomic clock still experiences noise and clock drift interference, and therefore, corrections need to be made. Corrections that need to be applied are stored in navigation files made with measurements (Enge \& Misra, 2006).

\subsection{User segment errors}

There are two types of user segment errors, and one is a receiver clock error, the other is a multipath error. Usually, direct line-of-sight measurements are accepted. However, the signal can also go through several paths, after being reflected on the surrounding objects or through the ground to the receiver. Multipath is longer than the direct line of sight to the satellite and therefore causes delays in both measurements (Enge \& Misra, 2001). Multipath signals can be characterized by their amplitude, and signals are usually weaker than direct signals, signal delay, phase, and phase rates of reflected signals concerning direct signals. When several waves come together, interference will occur, and multipath signals will change the signal directly (K. Yedukondalu, 2011).

\subsection{Atmospheric errors}

Because GPS signals travel through the atmosphere, they are influenced by the ionosphere and the troposphere, which causes two delays. This delay needs to be removed and corrected to find water vapor that can eventually occur.

Ionospheric delays can only be measured using measurements of dual-frequency GPS receivers. The ionosphere is an area that ranges from $50 \mathrm{~km}$ to about $1000 \mathrm{~km}$ above the earth's surface where free electrons and ions are located. Ionization is caused by solar radiation that causes refraction of the transmitted satellite signal. Because physical characteristics are very dependent on the Sun, the difference between day and night is very large. During the day, the molecules are broken down in ions and electrons, and thus the electron density increases. However, when the Sun has set, the ions and electrons recombine, reducing the electron density. The speed at which radio signals propagate depends on the number of electrons encountered when propagating to the medium. Therefore a parameter called 'total electron content' (TEC) is taken into account. TEC means the number of electrons found in a $1 \mathrm{~m} 2$ cross-section column extending from the satellite to the receiver. One way to eliminate ionosphere delays is to use a free combination of the ionosphere. Estimated delay of ionosphere groups at frequency L1 and frequency L2 using code measurements are:

$$
\begin{aligned}
& I_{\mathrm{L} 1}=\frac{f^{2} \mathrm{~L} 2}{f^{2} \mathrm{~L} 1-f^{2} \mathrm{~L} 2}\left(\rho_{\mathrm{L} 2}-\rho_{\mathrm{L} 1}\right) \\
& I_{\mathrm{L} 2}=\frac{f_{\mathrm{L} 1}}{f^{2} 1-f^{2} \mathrm{~L} 2}\left(\rho_{\mathrm{L} 2}-\rho_{\mathrm{L} 1}\right)
\end{aligned}
$$

Where IL1 and IL2 stand for ionospheric delays in the L1 and L2 frequency bands respectively, fL1 and fL2 stand for the L1 and L2 frequency band frequencies, stand 1 and stand2 stand for code measurements in the L1 and L2 bands respectively (Enge \& Misra, 2001).

Since TEC varies significantly in the equatorial region, and thus, the ionospheric delay, it is essential to determine this error very accurately (Enge \& Misra, 2006). Better insight into ionospheric variations in the Ugandan region is explained by A.M.Koning (Koning, 2016). The most suitable way of determining the ionospheric delay component is explained here as well. When the ionosphere delay is determined, it can be reduced from pseudorange measurements so that the ionosphere-free pseudorange is obtained.

The tropospheric delay consists of two layers: the hydrostatic component, mostly dependent on dry gas, and the wet component, which is dependent on water content. The previous component accounted for around $90 \%$ of the delay, but the variation in the delay was far greater in the wet delay because this component varied more spatially and temporally. Dry components can be modeled with millimeter level accuracy (Gabor, 1997). The dry and wet component together is a delay in the peak troposphere.

The dry component of the tropospheric zenith delay can also be called the Zenith Hydrostatic Delay (ZHD) and is estimated by models based on accurate pressure measurements at the GNSS station (Gabor, 1997). The wet component of the zenith troposphere is called the Zenith Wet Delay (ZWD). ZWD can be calculated by reducing ZHD from ZTD; the relationship between the two is as follows (K. Yedukondalu, 2011).

$$
Z T D=Z H D+Z W D
$$

In this study, we tried to learn how to get ZTD values using open source software, namely goGPS. Then we validate the ZTD value with the ZTD value generated from IGS along with UNR. In the last part, we analyze ZTD to find out the character of ZTD at certain times so that the value of ZTD can be used for meteorological purposes.

\section{Data and Method}

\subsection{Data set}

The main data that we use in this study is GNSS data in the Independent Exchange Receiver (RINEX) format. In the first station, we use the BAKO Station managed by BIG / BAKOSURTANAL and the second station we use the JOG2 station, which is managed by GFZ (Geo Forschungs Zentrum), the German Geo-research Center.

Epoch in this study, we use days of the year (DOY) $022-$ 025 / 22-25 in January, which represent the rainy season and DOY 230-233 coincide on August 17-20, which represents the 2018 dry season.

Next, we extract RINEX data to obtain troposphere data using sophisticated open-source GNSS software, called goGPS version 1.0 Beta from Geomatics Research and Development s.r.l. - Lomazzo, Italy (goGPS_MATLAB, 2018). 


\section{2.goGPS}

The main software for extracting RINEX data, namely goGPS. (Realini, 2009) goGPS is an open-source software application originally developed by the Realini (with contributions from the various thesis works by master students) since 2007 at the Geomatics Laboratory of Politecnico in Milano, Como Campus. It is specifically designed to improve the positioning accuracy of low-cost GPS devices by relative positioning and Kalman filtering. goGPS code was published online as free and open-source software in 2009; the project is open to collaborations since its publication it has received support and code contributions by users working in both academics and business companies from different countries (including Italy, Japan, Switzerland, Spain, and Germany). Strategies for processing RINEX data from goGPS, as below,

Constellation: GPS

Processing Technique: Precision Point Positioning (PPP) Cut off elevation: $7^{\circ}$

Processing rate: 5 MINUTES

Mapping functions: Niell mapping functions

Ionosphere modeling: iono-free

Dynamic model: static

Frequency: L1, L2

Type of observation: code and phase

Some of the research results using goGPS, among others, Realini et al. (2012) stated that goGPS could work in either real-time or post-processing, by getting raw GPS data in the input and providing position (i.e., coordinates) in the output. Herrera et al. (2016) goGPS published under a free and open-source license, goGPS can process data collected by any recipient but focuses on the treatment of observations by low-cost recipients. Realini et al. (2017) mentioned that goGPS could process and calculate the results of data acquisition from GNSS signal observations, which currently use smart devices compared to low-cost GNSS.

Gatti et al. (2018) Development of goGPS by including the estimated tropospheric delay, all algorithms and procedures needed to implement PPP (Precise Point Positioning) are implemented. goGPS software was based on a Kalman Filter estimation. In the last, it was reengineered from scratch, focusing on quasi-static (slowmoving) station processing and switching to multi-epoch least squares estimation using undifferenced multi-GNSS observations. Initially operating just in PPP, the new version was recently extended to process networks of GNSS stations in relative mode (Tagliaferro et al. 2019).

\subsection{Precision Point Positioning (PPP)}

The precise point positioning is used as a postprocessing method for determining the absolute position using stand-alone (thus un-differenced) GPS receivers. GPS PPP processing uses the ionosphere-free combinations of dual-frequency pseudorange and carrierphase observations.

The preference for PPP instead of DD processing arises from the benefit of processing each GPS station independently, and that simultaneous observation of the same satellite of the two stations are not needed (Yuan et al. 2014). The process of calculating tropospheric delay using goGPS, in the user interface display is equipped with the Precise Point Positioning (PPP) technique (Zumberge et al., 1997).

As a comparison and part of the validation of the ZTD values we obtained from goGPS, we used two international tropospheric products from International GNSS Services (IGS) with commercial software version 5 of the Bernese and University of Nevada Reno (UNR) with software from NASA Jet Propulsion Laboratory (JPL), namely GIPSY / OASIS II (Blewitt et al., 2018).

\section{Result and discussion}

3.1. ZTD differences, with and without troposphere delay gradient.

Based on the writings of Iwabuchi et al. (2003) that the determination of ZTD by considering the gradient delay troposphere has a significant difference in value with the determination of ZTD without using the gradient delay troposphere.

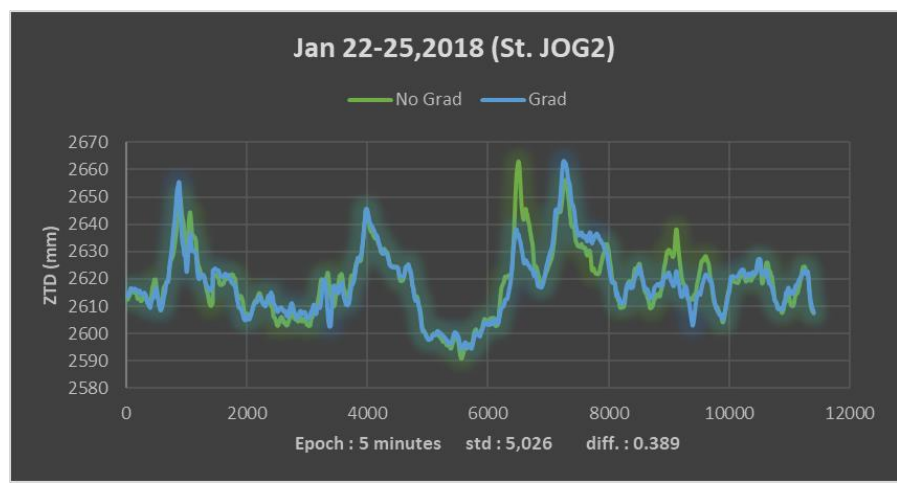

Figure3.1. The difference in the value of ZTD by using with and without troposphere delay gradient.

Therefore, the process of determining ZTD with software goGPS we consider the influence of the troposphere gradient delay. To prove this, we tried the ZTD determination process at the JOG2 station on January $22-25,2018$, our results for an average difference of up to 0.389 and the standard deviation were 5.026 as shown in the figure3.1.

The difference shows that there is a significant influence on the troposphere delay gradient on the ZTD value, therefore in this study of strategies in the process of determining ZTD with goGPS software, we use the troposphere delay gradient value.

\subsection{ZTD range value from goGPS}

First, we process data from BAKO and JOG2 stations from 22 to January 25, 2018. The data period that we process is every 5 minutes, so the amount of data for one day (24 hours) there are 288 data. From these 288 data, we get the smallest and largest and average values, as presented in Figure 3.2 as follows. 
Next, we carried out the same process from 18 to August 21, 2018. We present the results in Figure 3.3

ZTD values from 22 to January 25, 2018, in the BAKO station range tend to be higher than the range of ZTD values from 18 to August 21, 2018. Likewise, at the JOG2 station, ZTD range values from 22 to January 25, 2018, also show a higher average than ZTD range values are from 18 to August 21, 2018.

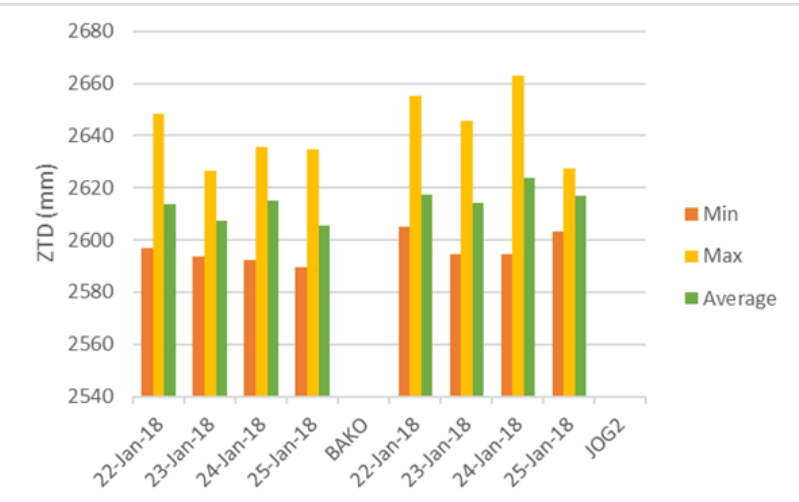

Figure3.2. Min, max and average values for 22-25 Jan 2018 at BAKO and JOG2 stations

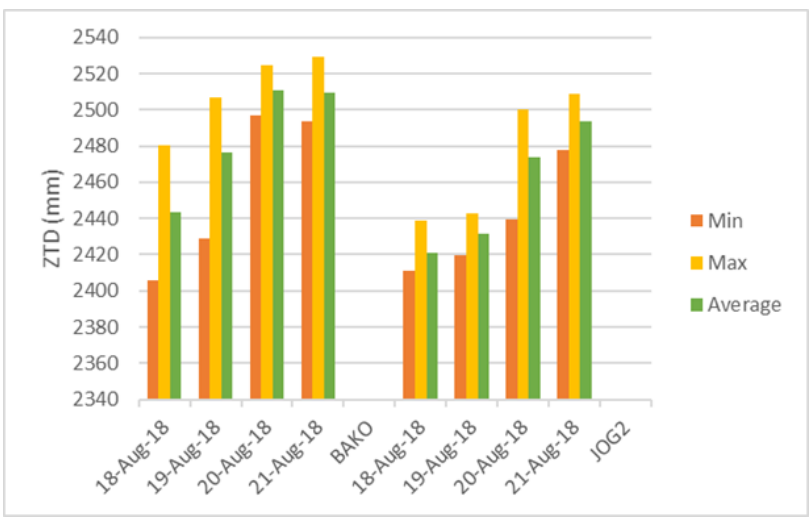

Figure3.3. Min, max and average values for 18-21

Aug 2018 at BAKO and JOG2 stations

Figure 3.2 and Figure 3.3 shows that there are differences in the value of ZTD depending on different time conditions, and this time difference is based on differences in seasons that exist in Indonesia, namely the rainy season and the dry season.

3.3. Standard deviation and Rms of the ZTD values of goGPS, IGS and UNR

Next, we determined the Rms values throughout the DOY, both at BAKO and JOG2 stations, which are part of the validation process of determining the ZTD value with goGPS.

Determination of the RMS value begins with determining the standard deviation values for each processing strategy, then the RMS values of the goGPS-IGS and goGPS-UNR are determined.

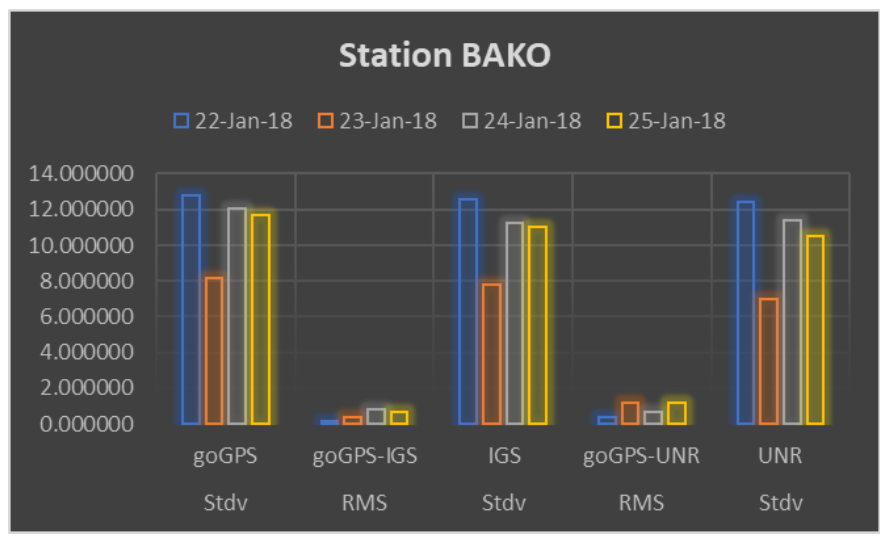

Figure3.4. Stdev and Rms for 22-25 Jan 2018 at BAKO stations

Our results show a small Rmsvalue of $<2 \mathrm{~mm}$. The RMS value is relatively small, meaning that the troposphere estimation process with goGPS shows a good agreement because it is almost the same as the international troposphere products from UNR and IGS.

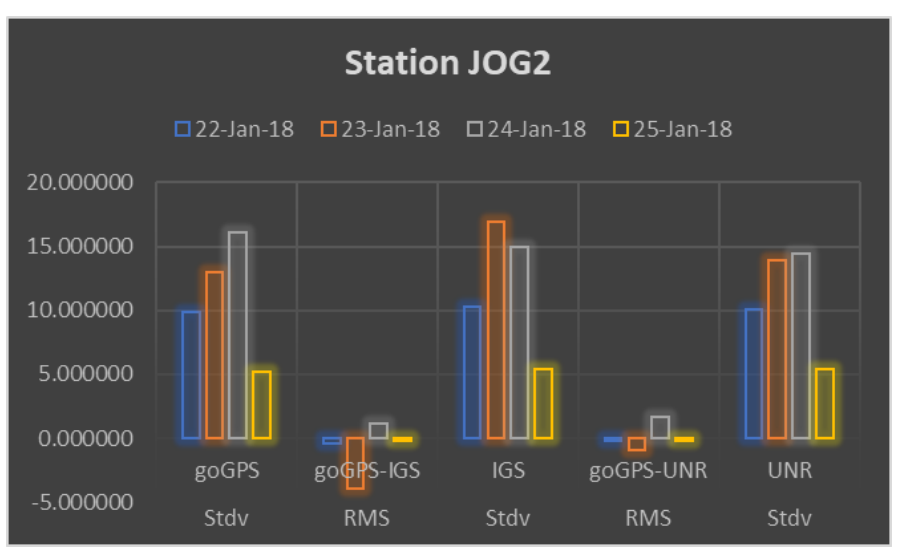

Figure3.5. Stdev and Rms for 22-25 Jan 2018 at JOG2 stations

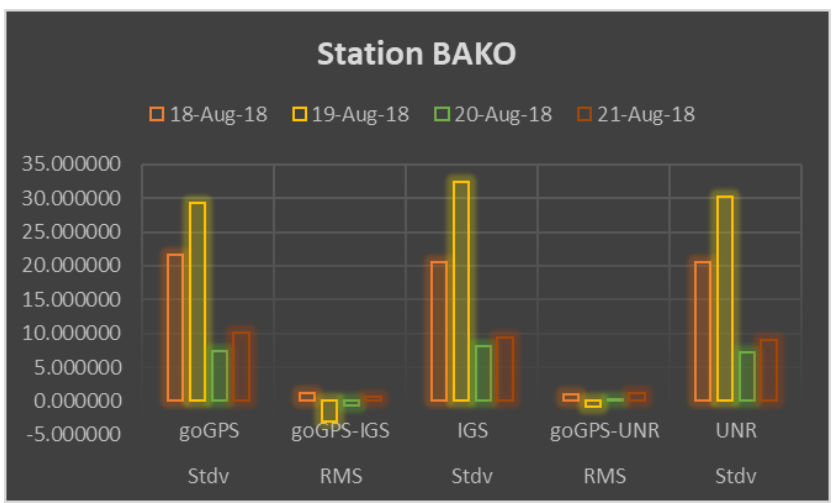

Figure3.6. Stdev and Rms for 18-21 Augt 2018 at BAKO stations 


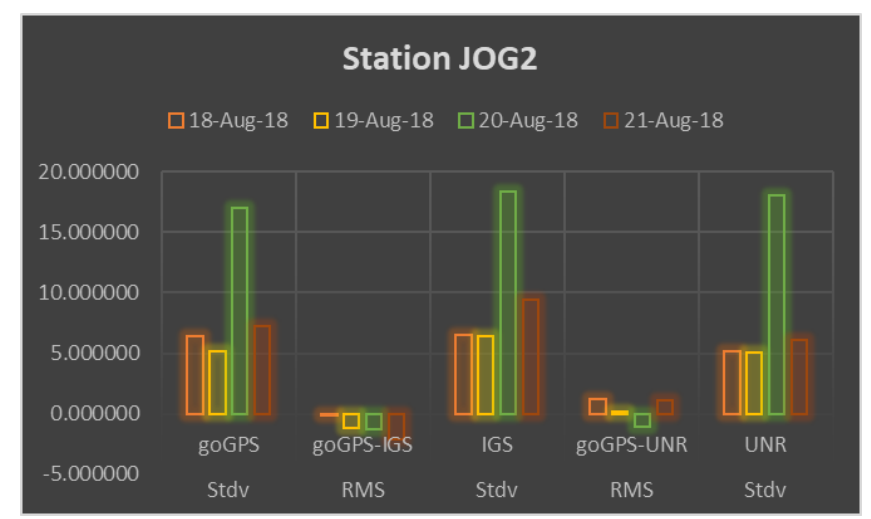

Figure3.7. Stdev and Rms for 18-21 Augt 2018 at JOG2 stations

The results of the ZTD values that we obtained using goGPS, both in January and August and the two BAKO and JOG2 stations, are shown in Figures 3.4 to 3.7. The whole picture shows the consistency of the ZTD value acquisition process with goGPS. GoGPS software works quite well at different times and stations. This means that the ZTD estimation process from goGPS software can be an alternative to paid software.

\subsection{Comparison of ZTD data for January and August}

After the validation process, goGPS can be an alternative determination of ZTD values. Then we carry out a full month ZTD estimation process to better see the relationship of ZTD values with differences in the January season representing the rainy season and August representing the dry season.

In January and August, compared to each in a separate Bako station and Jog2 station itself. The results are as in figures 3.8 and 3.9 .

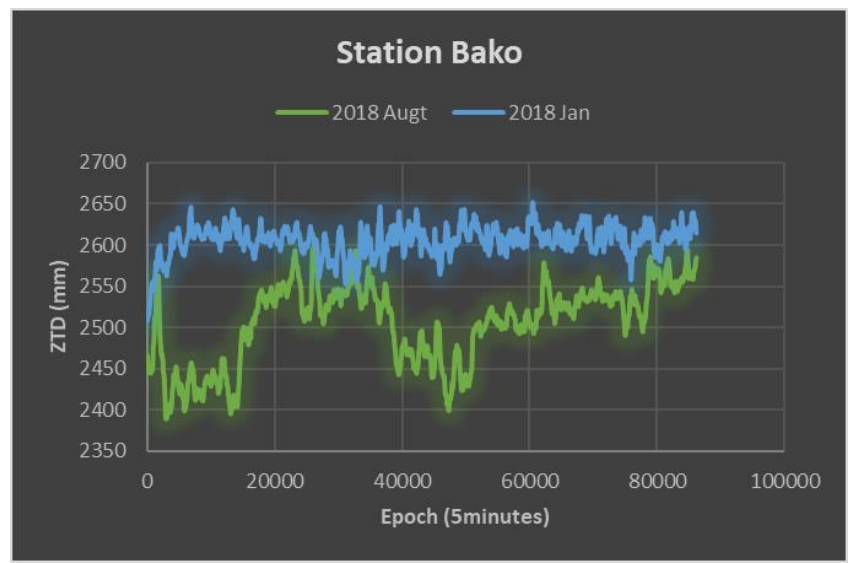

Figure3.8.ZTD Jan and Augt 2018 at BAKO stations

The figure shows that the tendency of ZTD value in January is higher than the value of ZTD in August for Bako and Jog2 stations, with an average difference of $99,632 \mathrm{~mm}$ and $142,602 \mathrm{~mm}$, respectively.

The condition of the ZTD value during January was relatively constant compared to August when there was a fluctuation in the value of the ZTD. This is seen in the standard deviation in August being greater due to fluctuations, compared to January, which was relatively stable. For BAKO station, the station is January $18,025 \mathrm{~mm}$, and August is $48,118 \mathrm{~mm}$. As for the JOG2 station, it was $22,986 \mathrm{~mm}$ in January and 42,988 $\mathrm{mm}$ in August.

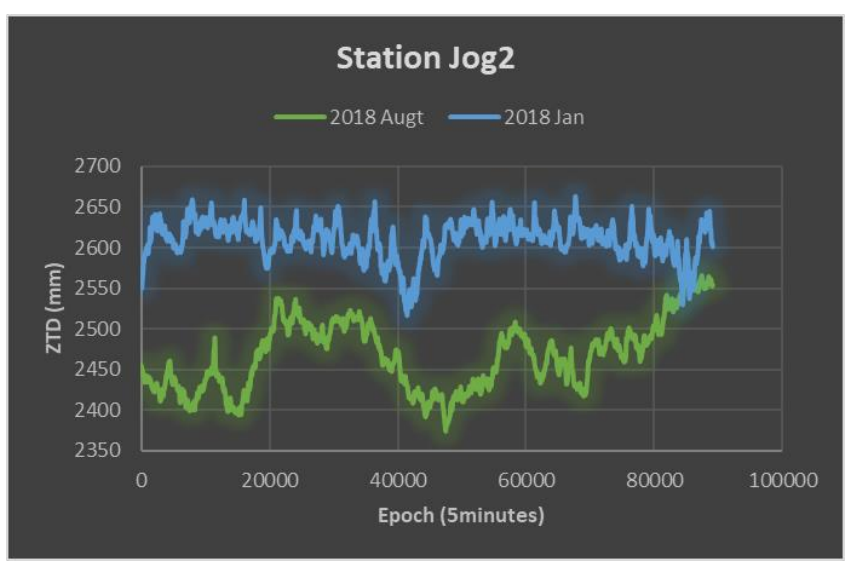

Figure3.9. ZTD Jan and Augt 2018 at JOG2 stations

This means that in the rainy season, the value of ZTD is higher than in the dry season, which shows that ZTD is an indication of conformity to the conditions of the rainy season and dry season; hopefully, this phenomenon can be more useful for meteorological purposes.

\section{Conclusion}

In this study, we used the open-source goGPS software to determine the ZTD value, which proved to be quite good when compared to other commercial software in determining the ZTD value.

ZTD values have characteristics in accordance with the conditions of changing seasons; in this case, when the rainy season, the value of ZTD tends to be high, and in the dry season, the value of ZTD is low, and fluctuations in ZTD values occur.

\section{Conflict of Interest}

The authors declare no competing interest.

\section{References}

Blewitt, G, W C Hammond, and C Kreemer. (2018). Harnessing the GPS data explosion for interdisciplinary science. EOS 99, DOI: 10.1029/2018E0104623.

Enge, P., \& Misra, P. (2001). Global Positioning System (1 ed.). Ganga-Jamuna Press.

Enge, P., \& Misra, P. (2006). Global Positioning System (2 ed.). Ganga-Jamuna Press.

Gabor, M. (1997, May 5). Remote Sensing of Water Vapor from GPS Receivers. Retrieved May 16, 2016, from http://www.csr.utexas.edu/texas_pwv/midterm/ gabor/gabor.html\#anchor561367.

Gatti, A., Tagliaferro, G., Realini, E. (2018). goGPS free and open-source GNSS software for tropospheric delay estimation. Proceedings from the 
conference held 4-13 April 2018 in Vienna, Austria, p. 15590

goGPS_MATLAB. (2018). goGPS MATLAB is an advanced GNSS observation processing software. goGPSproject. https://github.com/goGPSProject/goGPS_MATLAB (accessed April 2, 2018)

Herrera, A.M., Suhandri, H.F., Realini, E. et al. goGPS: opensource MATLAB software. GPS Solut 20, 595-603 (2016). https://doi.org/10.1007/s10291-0150469-x

K. Yedukondalu, A. S. (2011). Estimation and Mitigation of GPS Multipath Interference Using Adaptive Filtering. Progress in Electromagnetics Research $\mathrm{M}(21), 133-148$.

Koning, A. (2016). Precipitable water vapor estimation using GPS in Uganda: A study on obtaining the Zenith Wet Delay. Delft, The Netherlands

Nicholas, Z. (2016, October 04). Satellite and receiver clock errors. Retrieved from Tekmon Geomatics: http://www.tekmon.eu/1-3-2-satellite-andreceiver-clock-errors/

Realini, E. (2009) goGPS free and constrained relative kinematic positioning with low-cost receivers. Ph.
D.thesis,http://www.researchgate.net/publicatio $\mathrm{n} / 237520116$

Realini, E., Yoshida, D., Reguzzoni, M. et al. (2012). Enhanced satellite positioning as a web service with goGPS open-source software. Appl Geomat 4, 135-142 https://doi.org/10.1007/s12518-0120081-5

Realini, E., Caldera, S., Pertusini, L., Sampietro, D. (2017) Precise GNSS positioning using smart devices Sensors 17 (10), 2434

Tagliaferro, G., Gatti, A., Realini, E. (2019). goGPS Open Source GNSS Software for Quasi-Static Applications: Latest Developments and Performance Tests. Geophysical Research Abstracts. 2019, Vol. 21, p1-1.1p

Yuan, Y. K.-S. (2014). Real-time retrieval of precipitable water vapor from GPS precise point positioning. Journal of geophysical research: Atmospheres

Zumberge, J. F., Heftin, M. B., Jefferson, D. C., Watkins, M. M., and Webb, F. H. (1997). Precise point positioning for the efficient and robust analysis of GPS data from large networks. J. Geophys. Res. 102, 50055017. DOI: $10.1029 / 96 J B 03860$ 